\title{
Correction: Defining accelerometer cut-points for different intensity levels in motor-complete spinal cord injury
}

Tobias Holmlund · Elin Ekblom-Bak · Erika Franzén · Claes Hultling • Kerstin Wahman

Published online: 4 December 2019

(c) International Spinal Cord Society 2019

\section{Correction to: Spinal Cord}

https://doi.org/10.1038/s41393-019-0308-y

published online 26 June 2019

Following publication of this article, the authors noticed an error in the conclusion. The sentence: 'The cut-points for moderate-to-vigorous PA were defined as $\geq 7503$ for women and 9415 for men vector magnitude counts per minute (VMC) for PP and $\geq 4887 \mathrm{VMC} / \mathrm{min}$ for TP', has now been corrected to: 'The cut-points for moderate-to-vigorous physical activity were defined as $\geq 9515$ for PP and $\geq 4887$ $\mathrm{VMC} / \mathrm{min}$ for TP'. This has been corrected in both the PDF and HTML versions of the article. 